\title{
La démarche design, entre projet et expérience
}

Une poḯtique qui hybride penser et faire

\section{Estelle Berger}

\section{(2) OpenEdition}

\section{Journals}

Édition électronique

URL : http://journals.openedition.org/communicationorganisation/4714

DOI : 10.4000/communicationorganisation. 4714

ISBN : 979-10-300-0155-6

ISSN : 1775-3546

\section{Éditeur}

Presses universitaires de Bordeaux

\section{Édition imprimée}

Date de publication : 1 décembre 2014

Pagination : $33-42$

ISBN : 978-2-86781-905-6

ISSN : $1168-5549$

\section{Référence électronique}

Estelle Berger, "La démarche design, entre projet et expérience », Communication et organisation [En ligne], 46 | 2014, mis en ligne le 01 décembre 2017, consulté le 26 avril 2019. URL : http:// journals.openedition.org/communicationorganisation/4714; DOI : 10.4000/ communicationorganisation. 4714 


\section{La démarche design, entre projet et expérience Une poḯtique qui hybride penser et faire}

\section{Estelle Berger ${ }^{1}$}

La notion de design, activité de conception créatrice, a aujourd'hui pénétré tous les champs de notre vie, de l'objet utilitaire à l'expérience immatérielle. Mais, jusqu'ici, les théories qui s'y sont attachées lui ont rarement rendu justice. Si le design renvoie à des pratiques anciennes voire séculaires, il constitue une discipline jeune dont la formulation a pour une large part dépendu des définitions construites par des disciplines connexes, plus avancées dans la construction de leurs propres repères et concepts. Ainsi, aux yeux de l'architecture, le design n'est qu'une fraction circonscrite historiquement et en termes d'échelle. L'industrie, quand elle a reconnu le design, l'a soumis au fonctionnalisme et à la matérialisation de la technologie. Quant aux BeauxArts, ils ont tout au long du XXe siècle rejeté les arts appliqués et tenté de borner leur territoire de peur que celui-ci n'empiète sur le leur.

$\mathrm{Ne}$ peut-on pas dépasser ces querelles qui, en cloisonnant les pratiques, limitent les échanges et les hybridations fertiles ? Les designers, dont l'activité quotidienne comporte une facette intellectuelle largement mésestimée, ne sont-ils pas légitimes à prendre la parole ? Peut-on ainsi envisager des pensées du design émancipées de la mainmise d'autres disciplines ?

Il ne s'agit évidemment pas de rejeter en bloc les interventions de théoriciens extérieurs comme si elles constituaient une ingérence. Bien au contraire, les recherches menées par les sciences connexes fournissent des clés de compréhension sur la pratique, l'objet, la réception du design. Tout l'enjeu est plutôt d'articuler les apports de ces différentes disciplines, lorsque le point de vue adopté est propre au designer.

Cette problématique d'interdisciplinarité vaut autant au niveau théorique que pratique. Déjà dans l'exercice quotidien de son métier, le designer est à l'interface des acteurs d'un projet. Sans être ingénieur ni spécialiste des sciences humaines, il doit avoir une vision globale de ses paramètres, qui appartiennent

1 Estelle Berger est designer et chercheure (docteur en Arts Appliqués, équipe SEPPIA, Université de Toulouse Jean Jaurès), sur le fil entre profession et pensée du design. Sa démarche explore une poïétique du design comme pratique réflexive, qui se déploie entre expérience et discours ; estelle@yume-studio.fr 
tout autant à la technique qu'à la sociologie ou à l'anthropologie... Posséder une assise pluridisciplinaire permet ainsi au designer de comprendre ces éléments disparates, de les mettre en perspective, tout en exprimant sa vision spécifique et globale.

Rien de plus naturel alors qu'une épistémologie propre au design ne se développe ainsi, à la croisée des disciplines établies. Elle intègrerait, dans une préoccupation pragmatique, des outils théoriques comme pratiques empruntés aux différentes disciplines reliées au design. Cette approche n'estelle pas seule à même de développer une connaissance active, ancrée dans le réel, qui relierait praxis, pensées et implications sociales du design ? Elle apparaît comme un moyen de créer de la complémentarité là où il n'y avait que tension, et de dépasser les oppositions rhétoriques qui ont trop souvent cours entre théorie et pratique, académisme et terrain professionnel.

\section{Entre Arts et Sciences, mais ni art ni science}

La première dualité, entre raison et sensibilité, est vieille comme le monde, et semble particulièrement indépassable en France. Notre pays est à la fois vu comme celui de la gastronomie, du cinéma, du romantisme ; et comme celui de l'ingénierie ou de la recherche scientifique. Il a une légitimité dans ces deux univers, mais a étrangement créé une frontière étanche entre eux. En termes de design, cette opposition prend la forme de deux images archétypiques du concepteur. On aurait d'un côté un designer-artiste, qui exprime sa personnalité, son style à travers sa production, sans trop se soucier des contraintes de son client; et de l'autre un designer-ingénieur au service de l'industrie, dont le premier rôle serait d'imaginer de nouvelles applications des technologies pour les rendre acceptables.

Alors, artiste maudit ou artisan soumis ? Ces deux caricatures ne correspondent évidemment pas au quotidien du travail des designers, dont l'intervention est bien plus multifactorielle. Leur démarche n'est ni une recherche ordonnée de la vérité, ni le jaillissement de pulsions expressives. Elle est hybride, conciliant observation, compréhension du monde et action sur celui-ci. D'un côté une approche scientifique, de l'autre une vision d'ordre artistique. Ce que le design propose, c'est donc une médiation entre le monde de l'imaginaire et le sens de la réalité.

Cependant, s'ils dialoguent avec chacun des deux mondes et peuvent épisodiquement emprunter certaines de leurs méthodes, les designers n'appartiennent entièrement ni à l'un ni à l'autre.

Le design ne peut pas être défini comme une science, car c'est une activité projective et pas seulement de connaissance. Son point de vue se différencie en tant qu'il ne recherche pas d'invariants mais plutôt des singularités. L'objet d'étude scientifique, bien qu'il puisse être très précis, n'est jamais qu'un exemple prélevé dans une catégorie. Le design, au contraire, s'intéresse à la 
singularité de ses objets, à cette identité unique et contingente qui résiste à toute tentative de généralisation.

De plus, le design ne se contente pas de donner une interprétation du monde, il agit aussi sur lui de manière concrète en faisant advenir les objets et dispositifs qui constituent notre environnement. À l'inverse des sciences, il prend donc le monde comme sujet, et non comme simple objet d'étude. Pour Roger Nifle, un objet de recherche est une construction théorique au sein de laquelle on isole une hypothèse à tester et des variables à manipuler. La composante objet " marque la dimension d'altérité dans toute chose en tant qu'elle se distingue par séparation de son contexte " (Nifle, 1996 : 18). Au contraire, la composante sujet " marque la dimension d'intériorité, subjective, l'en-propre » (Nifle, 1996 : 18). D'un côté, la mise au jour de schémas existants, de l'autre, la création de formes au sein même du terrain étudié. C'est par le projet que les deux composantes s'articulent : "Objet et sujet ne sont pas dans un face à face, une opposition. Dans la trialectique sujet - objet - projet, la troisième dimension est le produit des précédentes. [...] Toute connaissance suppose intention subjective, conditions objectives et en final réalisation projective (rationnelle)»(Nifle, $1996: 18$ ).

La conception, projet du design, prend donc la forme d'une démarche raisonnée d'analyse - synthèse. Mais, dans ce processus, s'invite également un phénomène de transformation, de sublimation, plus mystérieux, par lequel l'ensemble des éléments raisonnés prend corps dans la mise en forme du projet, en dépassant la simple somme de leurs caractéristiques. L'artefact y puise son âme. Il ne s'agit pas de créer une œuvre comme dans un projet artistique, mais plutôt les conditions d'une expérience d'ordre esthétique. Cette dimension esthétique est essentielle dans le design, alors qu'elle ne fait pas partie de la mission de l'ingénieur par exemple, autre figure de la conception considérée sous un angle plus techniciste.

Néanmoins, qu'il relève de l'esthétique ne signifie pas que le design appartienne à la sphère artistique pour autant. D'emblée, ses moyens d'expression, pas seulement expressifs mais aussi cognitifs, l'en distinguent. Un lieu commun répandu voudrait que, comme celui d'un artiste, le travail d'un créatif soit un prolongement de sa personnalité - relevant de l'autobiographie plus que de la compréhension, du subjectif plus que de l'objectif. C'est certainement vrai pour certains designers-artistes, mais la grande majorité des designers contemporains revendique un travail d'analyse et de compréhension avant l'expression d'une personnalité. Cette recherche peut porter sur la problématique d'un client, l'amélioration de conditions existantes, une exploration d'un matériau pour ce qu'il est... En bref, l'utilisation dans sa pratique d'un schéma cognitif et non expressif. Contrairement à l'art, le design est fondamentalement autre chose que de l'expression de soi.

Entre l'art et le design, la différence réside peut-être dans un curseur qui mesurerait la liberté du praticien. Pour l'artiste, créer, c'est chercher en soi des 
réponses à des contraintes qu'il s'est imposées. Il est libre, mais seul responsable de ses problématiques et de sa production. Le designer, au contraire, manie des contraintes qui lui sont extérieures, et ne fait « que » recomposer des éléments préexistants mais disparates ou indéfinis, pour répondre à la problématique qui lui est soumise. "Que » entre guillemets car ce n'est pas parce qu'une liberté est plus relative qu'elle est plus simple à gérer. Le terme recomposer est important aussi, car en design, il ne s'agit pas d'inventer à partir de zéro - ce qui est plutôt un fantasme d'artiste, mais de transformer une situation subie en situation plus souhaitable. D'où $r e$ - pour indiquer qu'on ne crée pas au sens religieux du terme, c'est-à-dire en faisant surgir du néant. Et composer pour marquer la dimension intégrative du design, réunissant des éléments disparates dans un tout qui dépasse la simple somme de ses parties.

La démarche design emploie donc des éléments existants, qu'elle reconfigure selon des analogies imaginées dans l'esprit du designer, pour aboutir à un résultat inédit. Les pieds sur terre, la tête dans les étoiles, comme le suggère le dicton populaire.

\section{L'expérience}

Le designer s'appuie sur ses rencontres avec les autres, individus comme disciplines, pour construire sa démarche. Les intersections, les résonances, mais aussi les frictions participent tout autant à cette expérience. Chaque projet est ainsi l'occasion d'un bouleversement intime qui peut remettre en question toutes les certitudes. C'est cette force de l'expérience qui fait qu'un métier créatif est plus qu'un simple emploi, mais un choix de vie plus global où le renouvellement est perpétuel.

La notion d'expérience est envisagée ici dans une approche pragmatique, car la signification d'une expérience créative réside bien dans les caractéristiques de son vécu. Son apparition et sa forme se modulent en fonction du contexte. Finalement, on ne peut définir une expérience que par son influence sur un sujet précis et dans un contexte précis - c'est ce qui la rend si holistique et personnelle à chacun.

John Dewey s'est largement consacré à la théorisation du concept d'expérience, en philosophie comme en art. À son sens, elle constitue une manifestation esthétique, en tant qu'elle incorpore, dans une création unifiée et totale, toutes les dimensions du vécu - émotions, réflexions, souvenirs... L'expérience esthétique est donc créatrice, se manifestant en particulier dans la démarche de l'artiste ou du designer. Mais son accomplissement requiert des conditions particulières, un état de tension dynamique entre "l'union, l'intégration avec l'environnement », et « la conscience vivante » (Dewey, 2005: 80-81). Successivement, le sujet vivra donc des états d'immersion dans son environnement, et de perte de cette intégration à la vie ordinaire. L'expérience devient fertile justement par l'alternance de ces phases, qui permet à l'artiste vivant une expérience de formuler des objectifs et de mener à bien son projet. 
L'expérience esthétique du créateur est donc un processus conscient, d'intégration et d'accomplissement interne, fruit d'un mouvement organisé. Tout de même, elle laisse une large place à la contingence. Les idées sont des variations qui fluctuent, se propagent, selon des mécanismes qui échappent à la conscience. Le processus de création est donc une quête permanente qui alterne chaos, révolution, disruption, puis retour à un ordre nouveau qui stabilise et pérennise la proposition issue de ce mouvement. Jusqu'à la prochaine fois...

Forte de ce vécu expérientiel, la démarche design dépasse la dualité entre l'intelligible et le sensible, réunissant des pôles de l'esprit qui sont habituellement séparés. Par une démonstration concrète et intime au concepteur, elle réconcilie sensoriel, sensible et sensé.

On ne peut alors que dépasser la vision étriquée du design comme activité de conception visant à fournir une solution rationnelle à un problème. Il n'existe pas une seule bonne réponse, et de toute façon, le plus important est de formuler une bonne question. Comme un médecin, un designer ne peut se contenter de résoudre un symptôme pour soigner son patient. Le travail de diagnostic est prépondérant, et fait intervenir l'empathie plus que l'application de méthodologies. Pour adopter cette approche, il faut être capable de s'immerger dans le vécu, la pensée et le ressenti de l'autre, plutôt que de projeter sa propre vision ou une grille préétablie d'analyse du besoin à satisfaire.

Voilà pourquoi la pratique du design ne peut se limiter à l'application de méthodes. Le designer est une personne qui travaille pour d'autres personnes. Cette dimension humaine impose des limites à la planification et à la quantification, des moyens mis en œuvre comme des résultats attendus.

\section{La nécessité tactique}

Au sein d'une entreprise ou d'une organisation, le design est le seul organe qui non seulement admette, mais surtout tire parti de l'incertitude ambiante. Le marketing, les services commerciaux, financiers... cherchent au contraire à limiter l'imprévu, car ils craignent ses conséquences. Dans toute structure, une balance est nécessaire entre prévision et acceptation de la contingence. Un modèle fondé uniquement sur l'invention libre ne peut pas fonctionner concrètement, comme à l'inverse il est impossible d'éviter tout risque créatif. Réussir à équilibrer ces deux dimensions est un défi dans toute entreprise intégrant une dimension de création.

Le design peut et doit faire l'effort de s'adapter aux contraintes de l'entreprise - notamment en employant son langage, mais ce n'est pas toujours possible. La démarche de conception, si elle peut être modélisée schématiquement, ne peut être entièrement rationalisée. Les modèles et méthodes échouent souvent car ils véhiculent une vision linéaire du projet, en décalage avec la réalité. En termes de design et d'innovation, les approches prédictives sont inapplicables. 
Les entreprises n'ont pas d'autre choix que de l'admettre et de s'adapter au contexte dans lequel elles interviennent - un contexte fait d'incertitudes, de discontinuités, de disruptions. S'il y a encore souvent des réticences, il faut que les dirigeants intègrent qu'ils peuvent s'appuyer sur les designers, habitués à composer avec ces contraintes. Car la mission du designer consiste justement à réduire la complexité d'un système jusqu'à un niveau jugé acceptable, de transformer le chaos en un état plus sensé et compréhensible.

Répondre au prévisible comme à l'imprédictible, en milieu instable, demande d'adapter son schéma mental. L'intelligence se rend semblable à l'objet auquel elle s'applique, et si cet objet est un objet mouvant, qui prend toutes les formes, l'intelligence doit se faire elle-même plus polymorphe, plus souple et plus ambiguë que ce qu'elle essaye de connaître. Cette posture mentale, que réclame la démarche design, relève d'une métis - nom commun grec qui désigne une forme particulière d'intelligence faite de ruses, d'astuces, de stratagèmes, voire de dissimulations ou de mensonges. La métis était le modèle de l'opération rusée adoptée par les sophistes grecs, qui consistait à retourner contre un adversaire la puissance que celui-ci avait dirigée contre eux. Elle peut ainsi s'appliquer à un discours narratif comme à une pratique - plus particulièrement à une certaine catégorie de pratiques qui relèvent de la tactique. Ces formes d'anti-discipline, comme les nomme Michel de Certeau, renvoient à des arts de faire et à des savoirs tacites plutôt qu'à l'application de connaissances extérieures. Elles constituent ainsi une pensée qui ne se pense pas, qui ne se dit pas, et dont une part résistera toujours à l'explicitation :

La synthèse intellectuelle de la saisie d'éléments hétérogènes a pour forme non un discours mais la décision même, acte et manière de saisir l'occasion. [...] Le raté ou l'échec de la raison est précisément le point aveugle qui la fait accéder à une autre dimension, celle d'une pensée. Les pratiques fondées sur le rapport à l'occasion seraient donc dans la situation d'actes de pensée. Des gestes permanents de la pensée. [...] Si ce[s] «art[s]» ne peu[ven]t être que pratiqué[s], et que hors de [leur] exercice même il n'a pas d'énoncé, le langage doit en être aussi la pratique : un récit, à la fois art de dire et art de faire, théorie et pratique, condition et production. (Certeau, $1990: 101$ )

Effectivement, un projet n'existe pas dans l'absolu, seulement dans la réalité de sa pratique, dans son expérience. C'est pour cela que, si le design en tant qu'organe d'entreprise peut être considéré comme stratégique, en tant que démarche il relève plutôt de la tactique. Alors que la stratégie calcule les rapports de force et cherche à les maîtriser, la pratique tactique, elle, s'insinue et saisit des occasions. En termes de design, une posture stratégique correspond ainsi à livrer un produit utilitaire, inséré dans la logique commerciale d'une entreprise ; alors qu'une posture tactique prend plutôt la forme d'explorations, d'expérimentations, qui rusent, manipulent et détournent la technique ou d'autres contraintes rencontrées... Même s'il manie des connaissances à haute teneur intellectuelle, le designer ne travaille que sur des cas particuliers, 
en se guidant au gré des circonstances. C'est dans la pratique que s'accomplit l'essence de la discipline.

Mais le design est multidimensionnel, il est plus qu'une simple tactique qui consisterait à employer des outils définis pour obtenir un résultat anticipé. À chaque projet, les circonstances imposent de réinterroger toute la démarche, ses moyens et ses fins. Il faut se mettre à l'écoute de la contingence, articuler patience et réactivité pour agir lorsque le moment opportun se présente. Comme la figure grecque du Kairos dont il est difficile d'attraper la chevelure au passage, les occasions favorables peuvent facilement passer inaperçues. Savoir les saisir demande une attitude particulière, qui fait tout l'objet d'une démarche créative. Être créatif ne tient pas seulement d'une prédisposition, mais d'une attention permanente à se montrer réceptif à tout ce que l'on peut vivre ou percevoir. Ainsi peut-on trouver ce point, ce moment fertile où une action humaine délibérée vient rencontrer un processus naturel.

Il ne s'agit en fait de rien d'autre que de sérendipité. Ce terme, dérivé de l'anglais serendipity, a été inventé par l'écrivain Horace Walpole au milieu du XVIII siècle, inspiré par un conte d'origine persane, Voyages et aventures des trois princes de Serendip ${ }^{2}$, dans lequel les héros triomphent grâce à différentes découvertes qu'ils n'avaient pas recherchées. La sérendipité désigne donc une faculté de découvrir, d'inventer ou de créer sans préméditation, par sagacité accidentelle. L'élément surprenant peut se produire par hasard ou par erreur, peu importe tant que l'on en imagine une interprétation pertinente. La sérendipité est au cœur de toute découverte ; on lui doit nombre d'inventions célèbres comme les rayons $\mathrm{X}$ ou la pénicilline... Mais le mot n'est pas encore entré dans les dictionnaires français. Est-ce révélateur d'une incompréhension ou d'un manque de valorisation de la créativité, en sciences comme en arts? Cette posture active devrait au contraire trouver une place plus importante dans un monde professionnel qui réduit souvent les individus à une fonction et à une somme de tâches bien définies. Accepter le potentiel créateur de la sérendipité, c'est faire confiance à la pensée divergente des chercheurs et travailleurs créatifs - qui est féconde si on la laisse s'exprimer. Si les incertitudes sont accueillies avec compréhension, si le designer bénéficie de soutien, il peut alors prendre des risques et s'aventurer plus loin pour proposer des solutions non conventionnelles. À l'inverse, celui qui ne vise que des solutions parfaites et directement exploitables reste dans un cadre conventionnel qui risque de le censurer et d'étouffer sa force de proposition.

\footnotetext{
2 « Cette découverte est presque de l'espèce que j'appelle serendipity, un mot très expressif que je vais m'efforcer, faute d'avoir mieux à vous narrer, de vous expliquer : vous le comprendrez mieux par l'origine que par la définition. J'ai lu autrefois un conte de fées saugrenu, intitulé Les Trois Princes de Serendip: tandis que leurs altesses voyageaient, elles faisaient toute sorte de découvertes, par accident et sagacité, de choses qu'elles ne cherchaient pas du tout : par exemple, l'un des princes découvre qu'une mule borgne de l'œil droit vient de parcourir cette route, parce que l'herbe n'a été broutée que sur le côté gauche, où elle est moins belle qu'à droite - maintenant saisissez-vous le sens de serendipity? ». Horace Walpole, Lettre à son ami Horace Mann, 1754.
} 


\section{Conclusion : le designer comme passeur}

Pour lui laisser toute latitude à produire le meilleur, le monde de l'entreprise doit admettre que le design se nourrit de la contingence et lui est soumis.

Un bon management du design doit veiller à ce que tous les acteurs impliqués dans la démarche de conception soient placés sur un pied d'égalité, et communiquent de manière fluide en ayant la flexibilité d'esprit nécessaire pour se remettre en question lorsque la situation le demande. Il doit aussi résister contre la culture de l'urgence et du rendement qui règne dans certaines entreprises. Ne pas camper sur ses positions, et ne pas se précipiter - c'est à ce prix qu'une équipe peut se focaliser sur la réalisation d'un projet ou sur la mise en place d'une stratégie, en laissant de côté les préjugés et les luttes de pouvoir. Le dialogue entre métiers n'est pas toujours aisé, d'autant plus avec le design. Son langage technique et conceptuel et ses moyens d'expression spécifiques, passant souvent par l'image, ajoutent un filtre d'incompréhension avec certains interlocuteurs.

Mais les designers peuvent justement utiliser ce décalage pour jouer le rôle de passeurs entre humains et métiers. Ils ne sont pas des créateurs solitaires, mais membres d'une équipe réunissant, d'amont en aval, commanditaires, collaborateurs issus de tous univers, et usagers surtout. Car, finalement, c'est vis-à-vis de ces derniers qu'un projet prend tout son sens. Il n'est pas une simple affaire d'initiés, mais s'adresse avant tout à un public qui s'appropriera l'artefact créé. Le design n'est pas fait de concepts mais d'idées matérialisées dans des corps.

Avant d'être un organe d'entreprise, un métier ou une position hiérarchique, il est d'abord une interface entre les autres organes vitaux de tout projet de conception. Il a fallu du temps pour que ce rôle de médiateurs soit reconnu aux designers, mais les évolutions récentes de l'exercice du métier témoignent que, plus que praticiens, ceux-ci tendent à devenir des consultants. Le versant qui consiste à développer un projet et aboutir à un artefact devient ainsi moins important que la part plus immatérielle de l'intervention. Le design devient alors un moyen d'interroger un terrain, d'y révéler des problématiques, et de mettre en relation des disciplines et individus pas toujours habitués à travailler ensemble. Il agit comme aide à la décision, comme facilitateur. Activité transversale, le design se charge de récolter et d'assembler les éléments du puzzle, permettant à tous d'y voir plus clair, d'enrichir la réflexion et de démultiplier les capacités de tous les acteurs d'un projet.

Bien sûr, tout comme les cellules de recherche $\&$ développement ne sont pas seules à innover, le design ne peut revendiquer un monopole de la création. Mais sa mission diffuse, holistique, demande une implication en continu et une vision forte et ambitieuse dans les entreprises et collectivités pour donner la pleine mesure de son efficacité. Surtout, le design doit pouvoir investir des territoires encore indéfinis, s'inviter dans d'autres domaines disciplinaires. Car ce sont justement l'iconoclasme, la critique 
ou l'impertinence face aux idées reçues qui sont les meilleurs vecteurs de créativité et de pertinence des idées.

\title{
BIBLIOGRAPHIE
}

AMAR Georges (2005), « L'objet, entre design et prospective », in Actes du colloque Le design en question(s). Disponible sur : http://a.appliques.creteil.free.fr/telechargements/ design/AMAR\%20G.pdf

CHARAUDEAU Patrick (2010), « Pour une interdisciplinarité "focalisée" dans les sciences humaines et sociales ", Questions de Communication, n ${ }^{\circ}$ 17, p. 197-222.

CERTEAU Michel de (1990), L'invention du quotidien, tome 1 : Arts de faire, Paris, Gallimard (Folio essais).

DEWEY John (2005), L'art comme expérience, Paris, Gallimard. (Folio essais)

GAUDIN Thierry (1982,) "Société de création et épistémologie industrielle ", Culture technique, $\mathrm{n}^{\circ}$ 8. Disponible sur : http://hdl.handle.net/2042/30812

JOLLIVET Marcel, PENA-VEGA Alfredo (1998), « Relier les connaissances, le défi du $20^{\mathrm{e}}$ siècle ». In Compte-rendu des journées thématiques conçues et animées par Edgar Morin. Réed. Natures Sciences et Sociétés, 2002, Vol. 10, nº 1, p. 78-95.

JOLIOT Pierre (2001), La recherche passionnément, Paris, Odile Jacob.

LECHOT HIRT Lysiane (2010), Recherche-création en design. Modèles pour une pratique expérimentale, Genève, Métis Presses.

NIFLE Roger (1996), « La trialectique sujet objet projet; Les structures de l'expérience et de l'existence humaine », Le journal permanent de l'humanisme méthodologique, p. 18-35.

Résumé : Ni guide a priori, ni justification a posteriori, la démarche du design se construit chemin faisant, dans une poiétique personnelle qui fait intervenir la contingence au gré des déterminations comme des hasards qui jalonnent le projet. Démarche intellectuelle et sensible, elle hybride pensée et faire, et matérialise le double sens du mot conception - connaissance et création. Au cœur de cette dynamique du projet réside l'expérience du designer, qui incorpore, dans une création unifiée, toutes les dimensions du vécu. Par nature, la démarche design relève ainsi de la tactique plus que de la stratégie. À l'inverse des autres organes de l'entreprise, elle tire parti de l'imprévu. Elle concilie la sérendipité et la prévisibilité. Elle enrichit la culture du résultat d'une incitation à l'expérimentation. C'est cette posture singulière de médiation qui fait l'identité du design, qui ne pourrait se laisser réduire à un processus de conception rationnel sans perdre un peu de son âme. Mots-clés: poïétique, expérience, tactique, expérimentation, sérendipité, interdisciplinarité.

\begin{abstract}
Design approach neither relies on guides nor justifications, but builds itself on the way, as a personal poietics taking contingency into account throughout the project. Both an intellectual and sensitive process, it crosses thinking and doing, materializing the dual meaning of the word conception-knowledge and creation. Each project is a unified creation in which the designer embeds all dimensions of real-life experience. Thereby, the design process is by nature a matter of tactics more than strategy. Unlike any other organ of a company, it takes advantage
\end{abstract}




\section{C\&O n ${ }^{\circ} 46$}

of the unforeseen. It combines serendipity and predictability. A results-oriented culture benefits from its incentive to experiment. It is this posture of mediation that gives design its unique identity, adding a soul to a rational process of conception.

Keywords: poietics, experience, tactical intelligence, experimentation, serendipity, interdisciplinarity. 